\title{
Programa de robótica educativa para mejorar el aprendizaje significativo en estudiantes del cuarto grado del área de Ciencia y Ambiente de la institución educativa San Roque - Castrovirreyna, 2015.
}
Educational robotics program to improve meaningful learning in fourth grade students in the area of Science and Environment of the educational institution San Roque - Castrovirreyna, 2015

Dra. Aliaga Contreras, Isabel Margarita

Universidad Nacional del Centro del Perú

Dr. Carhuaricra Cusipuma, José Universidad Nacional de Huancavelica

Dra. Asencios Trujillo, Lida Violeta

Universidad Nacional de Educación Enrique Guzmán y Valle

Dra. Piñas Rivera, Livia Cristina

Universidad Nacional de Educación Enrique Guzmán y Valle

Recepción $10 / 05 / 2018$

Revisado $15 / 05 / 2018$

\section{Resumen}

71 presente artículo es la reseña de una investigación que tuvo como objetivo desarrollar un Programa de Robótica Educativa para mejorar el aprendizaje significativo del Área de Ciencia y Ambiente en estudiantes del cuarto grado de secundaria de la institución educativa San Roque -Castrovirreyna, 2015. El diseño de estudio fue cuasi experimental, la muestra 68 estudiantes. El instrumento aplicado para la medición del aprendizaje del área de Ciencia y Ambiente fue construido en base a la escala de actitudes de Likert de cinco puntos, validada por el criterio de jueces y una confiabilidad del $87 \%$. Los resultados muestran que el programa de robótica educativa influye de forma positiva y significativa en el aprendizaje del área de Ciencia y Ambiente; la intensidad de la influencia es del 55,9\% que resulta muy significativo.

Palabra clave: robótica educativa, Ciencia Ambiente, aprendizaje. 


\section{Summary}

7 he present article is the review of an investigation that had as objective to develop an Educational

1 Robotics Program to improve the significant learning of the Area of Science and Environment in fourth grade students of the educational institution San Roque -Castrovirreyna, 2015. The design It was quasiexperimental in a sample of 68 students. The applied instrument for the measurement of learning in the area of Science and Environment was constructed based on the Likert attitude scale of five points, validated by the criteria of judges and a reliability of $87 \%$. The results show that the educational robotics program has a positive and significant influence on learning in the area of Science and Environment; the intensity of the influence is $55.9 \%$, which is very significant.

Keyword: Educational robotics, environmental science, learning. 


\section{Introducción}

Determinado por el desarrollo social y el avance del conocimiento, se ha dado paso a la aparición de nuevas disciplinas que vinculan áreas científicas y tecnológicas de acrecentamiento de la productividad, la precisión y la calidad de nuevos productos y servicios disponibles para todas las áreas de la vida humana.

Como refiere Perú Educa (2016), la tecnología acompañó el desarrollo de la humanidad a través de la solución de problemas. Desde la rueda, aportó al hombre enormes posibilidades. La palanca, el plano inclinado, las poleas, los engranajes, etc. son máquinas simples que permiten desplegar una fuerza mayor que la que una persona podría aplicar solamente utilizando sus músculos o aplicarla de forma más eficaz y son algunos de los principios de la ciencia aplicados a la robótica educativa. En todo el universo del desarrollo tecnológico, probablemente, como uno de los resultados más importantes destaca la robótica, que desde su nacimiento se ha presentado como un medio de altas potencialidades de aplicación en las diversas áreas sociales y, con igual perspectiva, en el plano educativo. Inicialmente surgió en el seno de los mayores centros de producción mundial del conocimiento y como refiere Zárate (2013), los cambios tecnológicos, consecuencias del auge económico buscaron responder a la pregunta ¿cuál es el impacto de la robótica en la vida social? Una cuestión importante que ha inspirado en el campo educativo a preguntarse: ¿cuál es el impacto del Programa de Robótica Educativa en la mejora del aprendizaje significativo en el Área de Ciencia y Ambiente en estudiantes del cuarto grado de la institución educativa San Roque - Castrovirreyna, 2015? Responder esta pregunta en un contexto emergente, cambiante, signado de tecnología e información para el proceso educativo y de enseñanza aprendizaje siguen siendo fundamentales. El proceso de la globalización que implica todas las áreas del desarrollo social, a fin de cuentas es un desarrollo tecnológico multifactorial donde la robótica educativa constituye un recurso eficaz, para el trabajo interdisciplinario. La robótica ejerce mejora en el aprendizaje de los estudiantes, desarrollando sus competencias y capacidades de socialización, creatividad, liderazgo y trabajo colaborativo, que una vez aprendidas les permitirá plantear alternativas de solución a los problemas que se presenten en su contexto inmediato (Perú Educa, 2016). 
La robótica educativa está sustentada en las prácticas docentes, de acuerdo a las teorías filosóficas constructivistas de Piaget (1952) y Vygotsky (1978), así como el fundamento pedagógico del construccionismo de Papert (1993). Así, a mitades de los 80, miembros del equipo del Instituto Tecnológico de Massachusetts desarrollaron el LEGO TC Logo con el conocido juguete de construcción Lego. LOGOTC. Logo permite a los niños controlar las estructuras que construyen con Lego. Los niños programan la computadora para hacer que sus construcciones se muevan, hablen, se enciendan o respondan a diversos estímulos. Los “comportamientos" de estas máquinas pueden llegar a ser complejos. Lo interesante es que todo este desarrollo tiene sustento en los trabajos de diferentes psicólogos cognitivistas. Según Piaget (1952), en el proceso cognoscitivo, la asimilación y acomodación son dos aspectos, relacionados, facilitadores, del procesamiento de información y producción del conocimiento. En base al postulado de Piaget, Papert (1983) desarrolló la teoría del aprendizaje del construccionismo, basada en la acción, es decir, del proceder activo inspirada en las ideas de la psicología constructivista Papert postuló la defensa del lenguaje de programación trasladada a las aulas escolares para enseñar matemáticas a los niños. Otro factor importante del aprendizaje construccionista es que anima a los estudiantes a sacar sus propias conclusiones a través de la experimentación creativa y la elaboración de los objetos sociales. El docente constructivista se constituye en mediador. En lugar de adoptar una posición instructiva asume el papel de facilitador. La enseñanza se sustituye por la asistencia al estudiante en sus propios descubrimientos a través de construcciones que le permiten comprender y entender los problemas de una manera práctica. En ese sentido, el desarrollo de competencias básicas investigativas conlleva la explicación del mundo físico por medio del diseño y construcción de prototipos tecnológicos para resolver problemas de su entorno. El estudiante explora utilizando métodos científicos a situaciones, describe, explica los factores de la realidad en base a postulados científicos. Asimismo, al encontrarse con situaciones problemáticas de la realidad, ensaya el diseño y producción de modelos que a la postre constituirán prototipos tecnológicos para construir alternativas tecnológicas en aras de mejora de la calidad de vida.

Con el propósito de contribuir en mejores resultados del desarrollo tecnológico en el Perú, el Ministerio de Educación (2011) ha diseñado varios mecanismos de adquisición y equipamiento de medios y con ello la puesta en marcha de 
los Centros de Recursos Tecnológicos (CRT) de las instituciones públicas que a la postre buscan el desarrollo del pensamiento crítico y creativo de los estudiantes, la resolución de problemas, el desarrollo de destrezas en razonamiento matemático, la comprensión de textos, entre otros.

Otro factor fundamental, en aras del desarrollo tecnológico, fue la creación del Programa Nacional de Recuperación de las Instituciones Públicas Emblemáticas y Centenarias. Un aspecto particular es el otorgamiento de facultades al Ministerio de Educación para la adquisición de material didáctico tecnológico de robótica como máquinas simples iniciales, mecanismos motorizados, energías renovables y el kit de robótica "WE DO" con manuales para el docente, los cuales se organizan en los Centros de Recursos Tecnológicos (CRT). Asimismo, también el auge que se le otorga a dos aspectos fundamentales:

1. Programa de capacitación docente.

2. Supervisión y monitoreo: Realización de visitas de acompañamiento y monitoreo a cada centro de recurso tecnológico de las instituciones educativas emblemáticas, brindando asesoría a los docentes en la aplicación de los materiales didácticos en el desarrollo de sesiones de clase y a los coordinadores de CRT en el uso y manejo de los materiales didácticos.

Los centros de recursos tecnológicos deben enfatizar en el uso de materiales de robótica WE DO conjuntamente con la aplicación de las laptops XO para el desarrollo de sesiones de aprendizaje; el no aprovechamiento de la robótica como herramienta pedagógica podría limitar el interés de los estudiantes por las ciencias tecnológicas; en cambio, el trabajo en equipo, la práctica de valores, la creatividad, la acomodación, asimilación, etc. constituirán medios significativos de buenos aprendizajes.

\section{Objetivo general}

Desarrollar el Programa de Robótica Educativa para mejorar el aprendizaje significativo en estudiantes del cuarto grado del Área de Ciencia y Ambiente de la institución educativa San Roque - Castrovirreyna, 2015. 


\subsection{Objetivos específicos}

- Aplicar la tecnología para la adquisición de aprendizajes significativos relacionados al cuerpo y conservación de la salud, en los estudiantes del cuarto grado de secundaria de la institución educativa San Roque - Castrovirreyna, 2015.

- Aplicar el kit de robótica para conocer a los seres vivientes y conservar el medio ambiente, en los estudiantes del cuarto grado de secundaria de la institución educativa San Roque - Castrovirreyna, 2015.

- Desarrollar capacidades que identifiquen cambios que se producen en el mundo físico, en los estudiantes del cuarto grado de secundaria de la institución educativa San Roque - Castrovirreyna, 2015.

\section{Método}

El estudio corresponde al tipo de investigación aplicada en cuanto busca resolver un problema de la realidad por medio del programa de robótica educativa en el aprendizaje de la Ciencia y Ambiente. Según Sierra (2002) "en estos estudios se deben determinar y definir previamente las variables, luego se formulan hipótesis, las mismas que deben probarse por métodos estadísticos" (p. 42). Asimismo, el nivel de investigación es explicativo; según Sierra (2002) en el nivel explicativo las investigaciones buscan especificar las propiedades importantes de los hechos y fenómenos que son sometidos a una experimentación o trabajo de campo. Sobre esta base, el presente estudio busca explicar la relación causal entre las dos variables.

\subsection{Métodos específicos}

- La Observación. Se utilizará para el logro de los objetivos planteados en la investigación; la misma que exige un planteamiento de forma sistemática del problema (Meléndez, 2011).

- Inductivo. De acuerdo con Meléndez ( (2011) nos permitirá partir de la observación de fenómenos o situaciones particulares que enmarcan el problema de investigación y concluir proposiciones y a su vez, premisas que expliquen el aprendizaje del área de Ciencia y Ambiente. 
- Deductivo. De acuerdo con Meléndez (2011) permitirá que las verdades particulares contenidas en las verdades universales se vuelvan explícitas; es decir a partir de teorías o estudios generales del impacto del programa de robótica educativa se llegarán a determinar explicaciones particulares del aprendizaje del área de Ciencia y Ambiente.

- Analítico y Sintético. De acuerdo con Meléndez (2011) permitirá al investigador conocer la realidad en el área de Ciencia y Ambiente de los estudiantes del cuarto grado de secundaria.

\section{Diseño de la investigación}

- Tomando en cuenta a Campbell \& Stanley (1966) el término diseño se refiere al plan o estrategia concebida para obtener la información y clasifican en diseños experimentales y no experimentales.

- En su forma general la presente investigación tiene un diseño pre experimental con un solo grupo, cuya notación es:

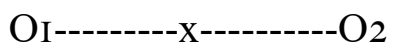

Dónde:

OI : Observación del pretest.

$\mathrm{O}_{2}$ : Observación del post test.

x : variable independiente.

\section{Muestra}

La muestra de estudio estuvo conformado 22 estudiantes del cuarto año de la Institución Educativa San Roque de la provincia de Castrovirreyna, período 2015, seleccionados mediante muestreo no probabilístico.

\section{Resultados}

De acuerdo al diseño del estudio, los resultados obtenidos en las fases del pre test y post test para ambos grupos son los siguientes: 
Dra. Aliaga Contreras, Isabel Margarita, Dr. Carhuaricra Cusipuma, José, Dra. Asencios Trujillo, Lida Violeta

Dra. Piñas Rivera, Livia Cristina

\section{Tabla 1}

Resultados del aprendizaje del área de Ciencia y Ambiente en el pre test

\begin{tabular}{|c|c|c|c|c|c|}
\hline \multirow{3}{*}{$\begin{array}{l}\text { Aprendizaje del } \\
\text { área de Ciencia y } \\
\text { ambiente }\end{array}$} & \multicolumn{4}{|c|}{ Grupo de estudio en el pre test } & \multirow{3}{*}{ Diferencia \% } \\
\hline & \multicolumn{2}{|c|}{ Control $^{\dagger}$} & \multicolumn{2}{|c|}{ Experimental ${ }^{\ddagger}$} & \\
\hline & $f$ & $\%$ & $\mathrm{f}$ & $\%$ & \\
\hline Inicio & 30 & 88,2 & 29 & 85,3 & 2,9 \\
\hline Proceso & 4 & 11,8 & 5 & 14,7 & 2,9 \\
\hline Logro & - & - & - & - & - \\
\hline \multirow[t]{2}{*}{ Total } & 34 & 100 & 34 & 100 & 0 \\
\hline & $\dagger \mathrm{m}=5,18$ & $\ddagger \mathrm{m}=5,43$ & \multicolumn{2}{|c|}{$|\mathrm{D}|=0,25^{*}$} & \\
\hline
\end{tabular}

*Sig. $=0,576$ [No significativa al nivel de 0,05 ]

Fuente: Cuestionario aplicado.

En la Tabla 1 se puede observar que al inicio del experimento los dos grupos en estudio son equivalentes, pues no existe diferencia significativa en las medias en lo referente al aprendizaje del área de Ciencia y Ambiente. El contraste de significancia obtenido es $p=0,576>0,05$. Estos resultados ponen en evidencia que ambos grupos al inicio del experimento están en condiciones iguales, por tanto, sus capacidades para diseñar y producir prototipos son las mismas. En esta fase, se arman grupos con los alumnos del grupo experimental y se expone la problemática a solucionar.

\section{Tabla 2}

Resultados del aprendizaje del área de Ciencia y Ambiente en el post test

\begin{tabular}{|c|c|c|c|c|c|c|}
\hline \multirow{3}{*}{$\begin{array}{l}\text { Aprendizaje del área de } \\
\text { Ciencia y ambiente }\end{array}$} & \multicolumn{5}{|c|}{ Grupo de estudio en el post test } & \multirow{3}{*}{$\begin{array}{c}\text { Diferencia } \\
\%\end{array}$} \\
\hline & \multicolumn{2}{|c|}{ Control $†$} & \multicolumn{2}{|c|}{ Experimental $\neq$} & & \\
\hline & $\mathrm{f}$ & $\%$ & $\mathrm{f}$ & $\%$ & & \\
\hline Inicio & 20 & 58,8 & 2 & 5,9 & - & 52,9 \\
\hline Proceso & 9 & 26,5 & 8 & 23,5 & - & 2,9 \\
\hline Logro & 5 & 14,7 & 24 & 70,6 & - & 55,9 \\
\hline Total & 34 & 100 & 34 & 100 & & 0 \\
\hline
\end{tabular}

*Sig. $=0,0$ [Significativa al nivel de 0,05$]$

Fuente: Cuestionario aplicado. 
En la Tabla, 2 se puede observar que al final del experimento los dos grupos en estudio tienen diferencias significativas, fundamentalmente, en el nivel de logro del aprendizaje del área de Ciencia y Ambiente. El contraste de significancia obtenido es $\mathrm{p}=0,0<0,05$ por lo que se concluye que el programa de robótica educativa ha tenido una influencia significativa en el aprendizaje. La intensidad de la influencia está dada, principalmente, en el nivel de logro de 55,9\%; que dentro del dominio probabilístico se tipifica como significativo. En el grupo experimental, se han elaborado dos proyectos, el primero de ellos, la construcción de un animal oriundo como la llama típica de nuestra región que nos provee carne y lana, la cual es muy suave y cuya fibra se usa para hacer vestimenta. Los estudiantes diseñan el prototipo y manipulan las partes de las piezas para construir el mismo, verifican el funcionamiento del prototipo y cada una de las partes. En el segundo proyecto, los estudiantes han elaborado un dispositivo para limpiar las carreteras, es decir, una excavadora; los estudiantes mejoran el diseño del primer proyecto y dibujan, realizan los cálculos necesarios y verifican su funcionamiento.

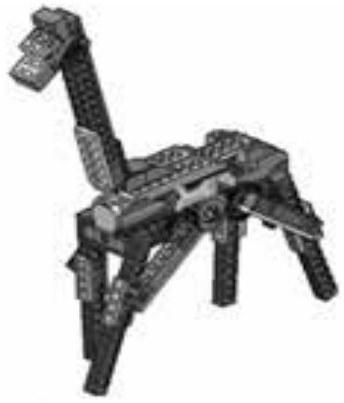

MAQUETA DEL PROYECTO 1

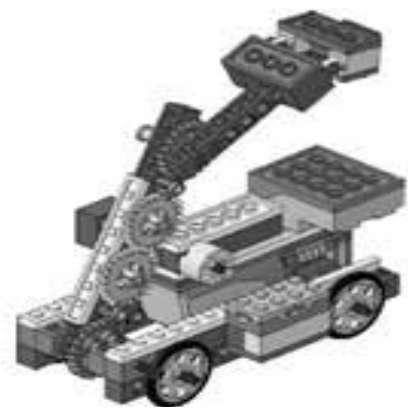

MAQUETA DEL PROYECTO 2 
Dra. Aliaga Contreras, Isabel Margarita, Dr. Carhuaricra Cusipuma, José, Dra. Asencios Trujillo, Lida Violeta

Dra. Piñas Rivera, Livia Cristina

\section{Tabla 3}

Resultados de logros obtenidos en el grupo control

\begin{tabular}{|c|c|c|c|c|c|c|}
\hline \multirow{3}{*}{$\begin{array}{l}\text { Aprendizaje del área de } \\
\text { Ciencia y ambiente }\end{array}$} & \multicolumn{5}{|c|}{ Grupo control } & \multirow{3}{*}{ Logro $\%$} \\
\hline & \multicolumn{2}{|c|}{ Pre test $†$} & \multicolumn{2}{|c|}{ Post test $\neq$} & & \\
\hline & $\mathrm{f}$ & $\%$ & $f$ & $\%$ & & \\
\hline Inicio & 30 & 88,2 & 20 & 58,8 & - & 29,4 \\
\hline Proceso & 4 & 11,8 & 9 & 26,5 & - & 14,7 \\
\hline Logro & - & - & 5 & 14,7 & - & 14,7 \\
\hline \multirow[t]{2}{*}{ Total } & 34 & 100 & 34 & 100 & & 0 \\
\hline & 5,18 & $\mathrm{~m}=5,58$ & \multicolumn{2}{|c|}{$|\mathrm{D}|=0,4^{*}$} & & \\
\hline
\end{tabular}

*Sig. $=0,164$ [No Significativa al nivel de 0,05 ]

Fuente: Cuestionario aplicado.

En la Tabla 3, se puede observar los logros obtenidos durante el experimento en el grupo control. El contraste obtenido es de $p=0,164>0,05$; por tanto, afirmamos que el grupo control no ha tenido un logro significativo, desde la fase del pre test al post test.

\section{Tabla 4}

Resultados de logros obtenidos en el grupo experimental

\begin{tabular}{|c|c|c|c|c|c|c|}
\hline \multirow{3}{*}{$\begin{array}{l}\text { Aprendizaje del área de } \\
\text { Ciencia y ambiente }\end{array}$} & \multicolumn{5}{|c|}{ Grupo experimental } & \multirow{3}{*}{ Logro \% } \\
\hline & \multicolumn{2}{|c|}{ Pre test $†$} & \multicolumn{2}{|c|}{ Post test $\ddagger$} & & \\
\hline & $\mathrm{f}$ & $\%$ & $f$ & $\%$ & & \\
\hline Inicio & 29 & 85,3 & 2 & 5,9 & - & 79,4 \\
\hline Proceso & 5 & 14,7 & 8 & 23,5 & - & 8,8 \\
\hline Logro & - & - & 24 & 70,6 & - & 70,6 \\
\hline \multirow[t]{2}{*}{ Total } & 34 & 100 & 34 & 100 & & 0 \\
\hline & $\dagger_{\mathrm{m}}=5,43$ & † $\mathrm{m}=8,43$ & \multicolumn{2}{|c|}{$|\mathrm{D}|=3,0^{*}$} & & \\
\hline
\end{tabular}

*Sig. $=0,0$ [Significativa al nivel de 0,05 ]

Fuente: Cuestionario. 
En la Tabla 4, se puede observar los logros obtenidos durante el experimento en el grupo experimental. El contraste obtenido es de $\mathrm{p}=0,0<0,05$; por tanto, afirmamos que el grupo experimental ha tenido un logro significativo desde la fase del pre test al post test. El logro obtenido es del 70,6\% que evidentemente es significativo, por lo cual, el programa de robótica educativa ha tenido un logro positivo.

El $n$ el grupo experimental, han trabajado en equipos, construyendo los dos modelos, explorando, investigando, escribiendo y debatiendo ideas que surgirán durante el uso de los modelos de dichas actividades. Estas actividades exigen criterios como orden lógico, exactitud y pone al alcance las herramientas, condiciones y tareas necesarias para llevar a cabo proyectos en distintos campos de aplicación.

Para la verificación de la hipótesis se ha utilizado la estadística " $t$ " de student para muestras independientes cuyo modelo es el siguiente:

$$
T=\frac{\mu_{e}-\mu_{c}}{\sqrt{\frac{S_{1}^{2}\left(n_{1}-1\right)+S_{2}^{2}\left(n_{2}-1\right)}{n_{1}+n_{2}-2}\left(\frac{1}{n_{1}}+\frac{1}{n_{2}}\right)}}
$$

Luego de aplicar los resultados se obtiene:

$$
T=\frac{8,43-5,58}{\sqrt{\left(\frac{1,61^{2}(34-1)+1,73^{2}(34-1)}{34+34-2}\right)\left(\frac{1}{34}+\frac{1}{34}\right)}}=7
$$

Utilizamos la función de la estadística " $t$ " para la toma de decisión de la hipótesis de investigación: 


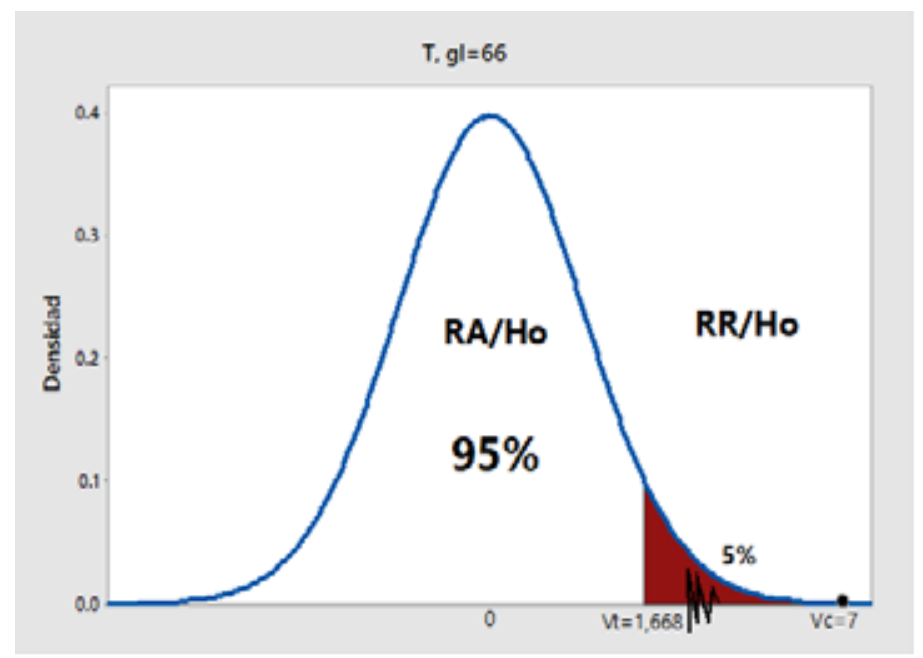

Figura 1. Distribución t para 66 grados de libertad.

Fuente: Software estadístico.

Por tanto, la decisión final es que el programa de robótica influye favorablemente en los aprendizajes significativos del área de Ciencia y Ambiente en estudiantes del cuarto grado de secundaria de la institución educativa San Roque - Castrovirreyna, 2015 con un 95\% de confianza.

\section{Discusión}

Los resultados del estudio son alentadores al propósito de lograr alternativas válidas para elevar los niveles de aprendizaje de estudiantes en la utilización de la tecnología en la educación a través de la aplicación de la robótica educativa. Para Hidalgo (2009) la robótica educativa es un medio de aprendizaje, en el cual participan las personas que tienen motivación por el diseño y construcción de creaciones propias. Estas creaciones se dan en primera instancia en forma mental y posteriormente en forma física, las cuales son construidas con diferentes tipos de materiales y controladas por un sistema computacional, los que son llamados prototipos o simulaciones. La robótica se constituye en un medio de acción disponible en los procesos educativos, por el carácter activo, participativo y cooperativo de los estudiantes, favoreciendo su evolución desde un punto de desarrollo cognitivo real a un punto de desarrollo cognitivo potencial, mediante la interacción social con sus pares y con el docente, consiguiendo superar sus zonas de desarrollo próximo. 
De acuerdo con Dengo (1998), la presencia de tecnologías en el aula de clase, busca proveer ambientes de aprendizaje interdisciplinarios donde los estudiantes adquieran habilidades para estructurar investigaciones y resolver problemas concretos, forjar personas con capacidad para desarrollar nuevas habilidades, nuevos conceptos y dar respuesta eficiente a los entornos cambiantes del mundo actual.

De la misma manera en la línea de MINEDU (2014), la Dirección General de tecnologías educativas del Ministerio de Educación promueve un proyecto nacional para integrar la Robótica Educativa en el contexto actual de la Educación Básica Regular de manera sencilla, articulada y flexible. Al momento de articularla con el Diseño Curricular Nacional, la robótica educativa se presenta como un medio de aprendizaje que busca la transversalidad curricular en diversas áreas. De esa manera, se puede aprovechar el aspecto multidisciplinario para aprender construyendo. De esta manera, los resultados obtenidos por la investigación corroboran las proyecciones del MINEDU.

\section{Conclusión}

1. La intensidad de la influencia identificada es del 55,9\% y la diferencia de medias identificada es de 2,85 unidades con un contraste de significancia asociado de $\mathrm{p}=0,0<0,05$. El 70,6\% de los casos tienen un nivel de logro en el aprendizaje del área de Ciencia Ambiente para el grupo experimental y solo un $14,7 \%$ para el grupo control.

2. El grupo control no presenta un logro significativo en el aprendizaje del área en referencia, el contraste obtenido es $p=0,164>0,05$. El grupo experimental presenta un logro significativo, pues, el contraste asociado es $\mathrm{p}=0,0<0,05$ lo que constituye la evidencia de la influencia del programa de robótica educativa.

3. La robótica se constituye en un medio de acción disponible en los procesos educativos, por el carácter activo, participativo y cooperativo de los estudiantes, favoreciendo su evolución desde un punto cognitivo real a un punto de desarrollo cognitivo potencial. 


\section{Referencias}

Cambridge, Ma.: Harvard University Press. Trad. Cast. de S. Furió (1979). El desarrollo de los procesos Psicológicos superiores. Barcelona: Crítica.

Dengo, O. (1998). La robótica pedagógica en los procesos de enseñanza y aprendizaje de las Matemáticas. Lima - Perú

García, A. (2003). Medios Informáticos. Buenos Aires: Kapeluz.

CAFAC(2011). Robótica Pedagógica y Robótica Educativa: características $y$ diferencias. Lima: KAF

Hidalgo, M. \& Martínez A. (2009). Control y Robótica Educativa en Tecnología. México: RIDU

Ministerio de Educación (2011). Robótica Educativa. Lima: WEDO

Ministerio de Educación (2011). Robótica en instituciones educativas de primaria multigrado. Perú: Educa. Recuperado de http://www. perueduca.pe/docentes/noticias/robotica-instituciones

Ministerio de Educación(2014). DIGETE presta asistencia técnica a proyectos educativos con tic a gobierno regional de Huancavelica. Recuperado de http://www.minedu.gob.pe/noticias/regiones.php?id=525 [Consultado 09 de agosto del 2018]

Odórico,A.(2005). LaRobótica desde una perspectivapedagógica. Recuperado de http://laboratorios.fi.uba.ar/lie/RevistaArticulos/020205A4ago2005. pdf [Consultado 09 de agosto del 2018]

Papert, S. (1980). Mindstorms: Children, computers, and powerful ideas. New York: Basic Books.

Papert, S. (1993). Mindstorms: Children, computers, and powerful ideas (2nd ed.). New York: Basic Books.

Perú Educa (2018). Robótica Educativa. Recuperado de http://www. perueduca.pe/robotica/ [Consultado 09 de agosto del 2018] 
Piaget, J. (1952). The Origins of Intelligence in Childhood - International Universities Press.

Ruiz, V. (2002). Ciencia y Tecnología a través de la Robótica Cognoscitiva. Centro de Estudios sobre la Universidad (CESU).. México: UNAM

Sánchez, C. (2003). Implementación de Estrategias de Robótica Pedagógica en las Instituciones Educativas. México: F.R.B.A

Sánchez, H. \& Reyes C. (1996). Metodología y Diseños en la Investigación Cientifica. Lima: Los Jazmines.

Universidad César Vallejo (2009). Diseño y desarrollo del trabajo de investigación. Escuela de Postgrado. Maestría en Administración de la Educación Trujillo Perú.

Vygotski, L. (1978). Mind in society. The development of higher psychological process.Ecuador: UCC.

Zárate, R. (2013). Nuevo Equipamiento. Recuperado de http://www. eldebate.com.ar/nuevo-equipamiento-la-tecnica-no-3-de-zarate-fueelegida-como-escuela-del-futuro-y-como-tal-recibio-kit-de-robotica/ [Consultado 09 de agosto del 2018] 